Few-Body Systems 0, 1-5 (2018)

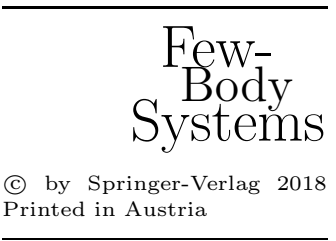

\title{
Two-boson Correlations in Various One-dimensional Traps*
}

\author{
A. Okopińska **, P. Kościk *** \\ Institute of Physics, University of Humanities and Sciences, Świętokrzyska 15, 25-406 Kielce, \\ Poland
}

\begin{abstract}
A one-dimensional system of two trapped bosons which interact through a contact potential is studied using the optimized configuration interaction method. The rapid convergence of the method is demonstrated for trapping potentials of convex and non-convex shapes. The energy spectra, as well as natural orbitals and their occupation numbers are determined in function of the inter-boson interaction strength. Entanglement characteristics are discussed in dependence on the shape of the confining potential.
\end{abstract}

\section{Introduction}

Entanglement as a measure of quantum correlations is investigated in the hope of better understanding the structure of strongly-coupled many-body systems. Recently there is a growing interest in studying few-particle trapped systems, since they became accessible in experiments with ultracold gases in optical lattices and microtraps. The interatomic interaction can be there considered as a contact one. By choosing the transverse confinement much stronger than the longitudinal one, the quasi-one-dimensional systems with an effective interaction $g_{1 D} \delta\left(x_{2}-x_{1}\right)$ of an adjustable strength $g_{1 D}$ may be experimentally realized [1]. In the Tonks-Girardeau (TG) limit of $g_{1 D} \rightarrow \infty$ the system is solvable for arbitrary trapping potential [2]. Theoretical consideration of such a system evolution from weak to strong interactions is thus of interest.

We discuss entanglement properties for a system of two bosons interacting through a contact potential and subject to a confining potential $V(x)$. The dimensionless Schrödinger equation takes a form

$$
H \phi\left(x_{1}, x_{2}\right)=E \phi\left(x_{1}, x_{2}\right),
$$

\footnotetext{
*Article based on the presentation by A. Okopińska at the Fifth Workshop on Critical Stability, Erice, Sicily, Received November 30, 2008; Accepted January 8, 2009

**E-mail address: okopin@fuw.edu.pl

${ }^{* * *}$ E-mail address: koscik@pu.kielce.pl
} 
where the Hamiltonian reads

$$
H=-\frac{1}{2} \frac{\partial^{2}}{\partial x_{1}^{2}}-\frac{1}{2} \frac{\partial^{2}}{\partial x_{2}^{2}}+V\left(x_{1}\right)+V\left(x_{2}\right)+g_{1 D} \delta\left(x_{2}-x_{1}\right)
$$

Since the two-boson function is symmetric and may be chosen real, there exists an orthonormal real basis $\left\{v_{l}\right\}$ such that

$$
\phi\left(x_{1}, x_{2}\right)=\sum_{l} k_{l} v_{l}\left(x_{1}\right) v_{l}\left(x_{2}\right)
$$

where the coefficients $k_{l}$ are real and $\sum_{l} k_{l}^{2}=1$. Therefore

$$
\int_{-\infty}^{\infty} \phi\left(x, x^{\prime}\right) v_{l}\left(x^{\prime}\right) d x^{\prime}=k_{l} v_{l}(x)
$$

which means that $v_{l}$ are eigenvectors of the two-particle function. It may be shown that $v_{l}$ are also eigenvectors of the density matrix, known as natural orbitals. Density matrix decomposition is given by $\rho\left(x, x^{\prime}\right)=\sum \lambda_{l} v_{l}(x) v_{l}\left(x^{\prime}\right)$, where the occupancies $\lambda_{l}=k_{l}^{2}$. The number of nonzero coefficients $k_{l}$ and the distribution of their values characterize the degree of entanglement.

\section{Optimized Configuration Interaction Method}

The configuration interaction method (CI) consists in choosing the orthogonal basis set in the Rayleigh-Ritz (RR) procedure so as to ensure proper symmetry under exchange of particles [3]. For the two-boson system, the CI expansion reads

$$
\phi\left(x_{1}, x_{2}\right)=\sum a_{i j} \psi_{i j}\left(x_{1}, x_{2}\right)
$$

where $\left\langle x_{1}, x_{2} \mid i j\right\rangle=\psi_{i j}\left(x_{1}, x_{2}\right)=b_{i j}\left[\varphi_{i}\left(x_{1}\right) \varphi_{j}\left(x_{2}\right)+\varphi_{j}\left(x_{1}\right) \varphi_{i}\left(x_{2}\right)\right]$ with $b_{i j}=1 / 2$ for $i=j$ and $b_{i j}=1 / \sqrt{2}$ for $i \neq j$. Exact diagonalization of the infinite Hamiltonian matrix $H_{n m i j}=\langle n m|H| i j\rangle$ determines the whole spectrum of the system. Truncated matrices $[H]_{N \times N}$ allow determination of successive approximations to the larger and larger number of states by increasing the order $N$. We use the one-particle basis of the harmonic oscillator eigenfunctions

$$
\varphi_{i}^{\Omega}(x)=\left(\frac{\sqrt{\Omega}}{\sqrt{\pi} 2^{i} i !}\right)^{\frac{1}{2}} H_{i}(\sqrt{\Omega} x) \exp \left[-\Omega x^{2} / 2\right] .
$$

Following the optimized RR scheme [4], we adjust the value of the frequency $\Omega$ so as to make stationary the approximate sum of $N$ bound-state energies, by requiring

$$
\frac{\delta \operatorname{Tr}[H]_{N \times N}}{\delta \Omega}=0 .
$$

Such a way of proceeding has been shown to improve strongly the convergence of the RR method [4, 5]. The $N$ th order calculation provides approximations to many eigenstates, which enables a direct determination of natural orbitals by 
representing them in the same basis (6) as $v(x)=\sum p_{n} \varphi_{n}^{\Omega}(x)$. This turns the eigenequation (4) into an algebraic problem

$$
\begin{aligned}
& \sum\left(A_{m n}-k_{n} \delta_{m n}\right) p_{n}=0, \\
& A_{m n}=\int \psi_{m}^{\Omega}\left(x_{1}\right) \phi\left(x_{1}, x_{2}\right) \psi_{n}^{\Omega}\left(x_{1}\right) d x_{1} d x_{2}=\left\{\begin{array}{cll}
a_{n n} & \text { for } & m=n \\
2^{-1 / 2} a_{m n} & \text { for } & m \neq n
\end{array}\right.
\end{aligned}
$$

and $a_{n m}$ are determined from diagonalization of $[H]_{N \times N}$. By diagonalization of the matrix $[A]_{N \times N}$, the approximate coefficients $k_{n}$ may be determined. Due to the fact that $\sum A_{n m}^{2}=1$, their numerical values satisfy $\sum k_{n}^{2}=1$.

\section{Results}

In the case of harmonic confinement $V(x)=m x^{2} / 2$ and the contact interaction, the two-particle wave function may be analytically expressed [6]. This allows determination of the occupancies $\lambda_{i}=k_{i}^{2}$ by discretizing (41). The two largest occupancies for the ground state are shown in Fig. 1 in function of $g_{1 D}$. The

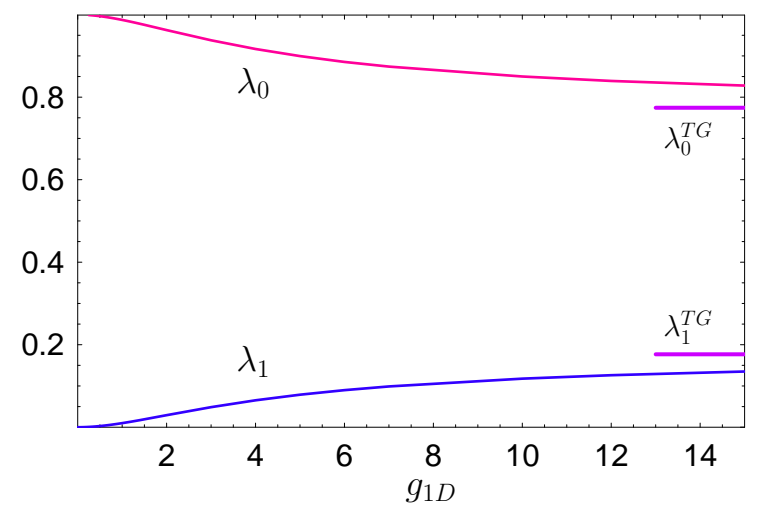

Figure 1. The occupancies $\lambda_{0}$ and $\lambda_{1}$ for a harmonically confined two-boson system in function of $g_{1 D}$, their TG limits are marked by horizontal lines.

state is non-entangled $\left(\lambda_{0}=1\right)$ only if the bosons do not interact. The weakly entangled "condensed" state with only one orbital significantly occupied is realized at very weak interactions, $g_{1 D} \lesssim 0.1$. With increasing $g_{1 D}$, the entanglement grows, which shows up in the increase of $\lambda_{1}$ at the cost of $\lambda_{0}$. The occupancies monotonically approach their TG limits $\lambda_{0}^{T G} \approx 0.7745$ and $\lambda_{1}^{T G} \approx 0.1765$.

Entanglement properties in the case of multi-well potentials are markedly different. Using the optimized RR method, we calculated the natural orbital occupancies of ground states in double-well potential $V_{2 \text { well }}(x)=\frac{2}{27 a}\left(1-a x^{2}\right)^{2}$ and triple-well potential $V_{3 \text { well }}(x)=\frac{1}{2} x^{2}-a x^{4}+\frac{a^{2}}{2} x^{6}$. The potentials have minima of the same depth and the maxima of the same height, controlled by the parameter $a$. The results for $a=0.025$ are plotted in Figs. 2 and 3, where the upper left presents the shapes of the potentials, and the lower left, the two largest occupancies $\lambda_{0}$ and $\lambda_{1}$ in function of $g_{1 D}$. For $g_{1 D}=0$, the ground state is nonentangled, as $\lambda_{0}=1$. With increasing interactions, $\lambda_{0}$ decreases and $\lambda_{1}$ grows, 

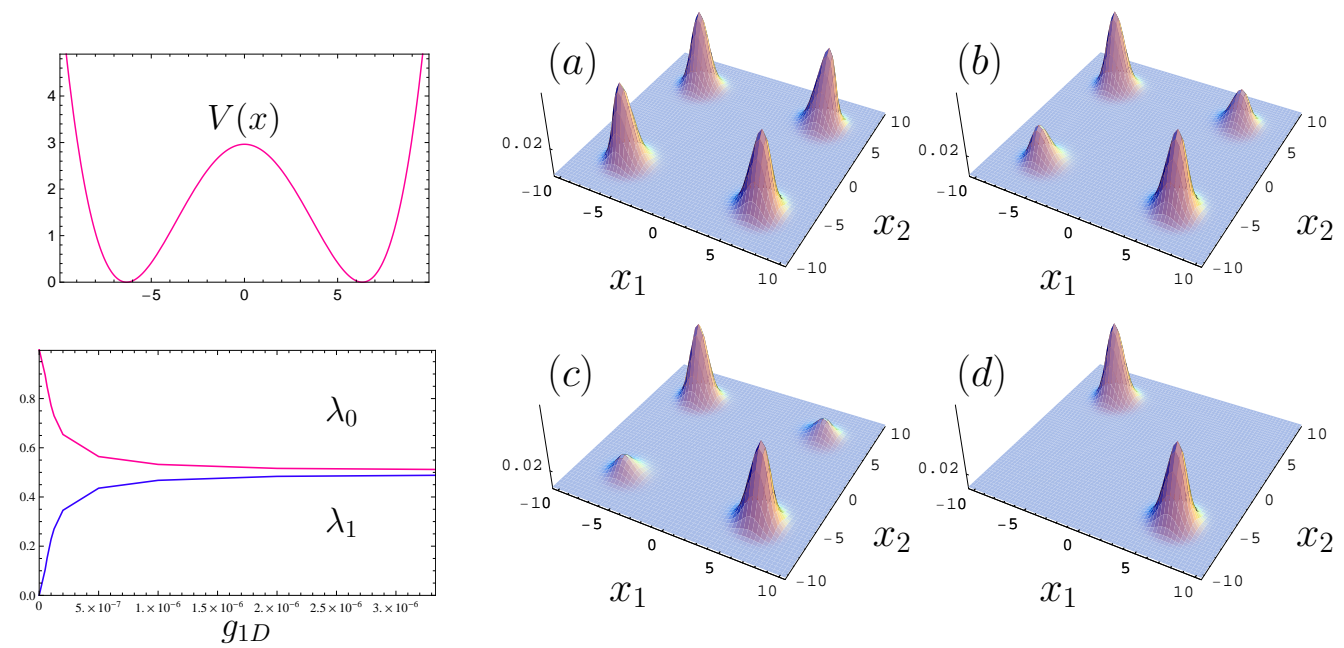

Figure 2. Double-well potential (upper left), the occupancies $\lambda_{0}$ and $\lambda_{1}$ in function of $g_{1 D}$ (lower left) and two-boson densities (right) for (a) $g_{1 D}=0$, (b) $g_{1 D}=2.5 \cdot 10^{-8}$, (c) $g_{1 D}=$ $5 \cdot 10^{-8},(\mathrm{~d}) g_{1 D}=10^{-6}$

monotonically approaching the TG limit of non-entangled "fragmented" state, $\lambda_{0}^{T G}=\lambda_{1}^{T G}=0.5$. The critical value $g_{1 D}^{c r}$, above which $\lambda_{0} \approx \lambda_{1}$, is much larger for the triple-well potential than for the double-well one. The dependence of the two-boson density on $g_{1 D}$ for the double-well potential is shown on the right of Fig. 2, For noninteracting bosons, the probability of both being in different wells is the same as being in the same well. With increasing $g_{1 D}$, the probability of finding the bosons in the same well quickly decreases and above $g_{1 D}^{c r}$ the state is almost fragmented. In the triple-well case (right of Fig. 3) the particles live in the middle well, only above $g_{1 D}^{c r}$ the probability of finding a particle in an external well becomes considerable. In the TG limit of non-entangled "fragmented" state, one particle is localized in the middle and the other in one of external wells. 

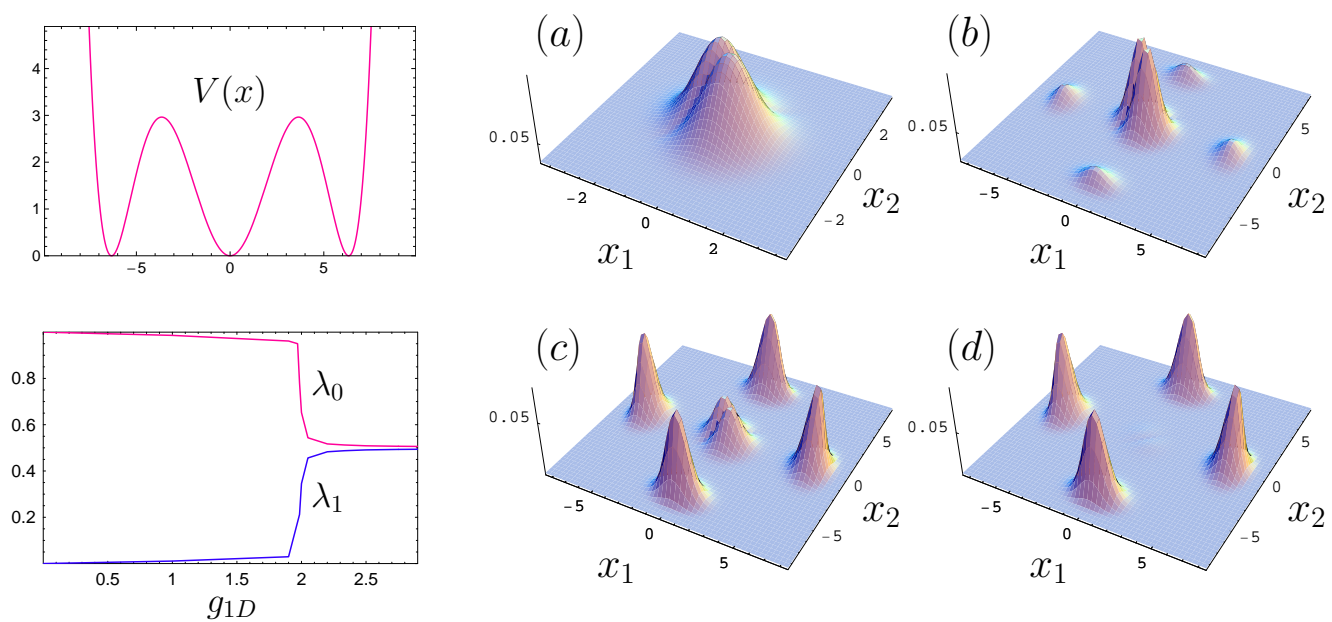

Figure 3. Same as Fig 2 but for the triple-well potential. The two-boson densities (right) for (a) $g_{1 D}=1$, (b) $g_{1 D}=1.97$, (c) $g_{1 D}=1.985$, (d) $g_{1 D}=2.05$

\section{Conclusion}

The optimized CI method proves very effective in determining the spectrum and the natural orbitals of the two-particle confined systems.

\section{References}

1. Kinoshita, T., Wenger, T., Weiss, D. S.: Science 305, 1125 (2004).

2. Girardeau, M.: J.Math.Phys. 1, 516 (1960)

3. Helgaker, T., Jørgensen P., Olsen J.: Molecular Electronic- Structure Theory (Wiley, Chichester, 2000).

4. Okopińska, A.: Phys.Rev. D36, 1273 (1987)

5. Kościk, P., Okopińska, A.: J. Phys. A: Math. Theor. 40, 10851 (2007)

6. Busch, T., et al.: Found. Phys. 28, 549 (1998) 